\title{
Charge Compensation for Imaging Large Insulating Samples By Using Secondary Ion Tandem Mass Spectrometry
}

\author{
R. T. Short, John M. McMahon, W. M. Holland, and Peter J. Todd \\ Oak Ridge National Laboratory, Oak Ridge, Tennessee, USA
}

\begin{abstract}
A charge compensation technique has been developed for secondary ion mass spectrometry and imaging of insulating samples as large as $1 \mathrm{~cm}^{2}$ using a triple quadrupole-based microprobe. The microprobe secondary ion extraction field is synchronized with a periodic primary $\mathrm{Cs}^{+}$beam to allow a sheetlike beam of $5-\mathrm{eV}$ electrons to pass over the sample surface when the extraction field is zeroed. Electrons are attracted to, and neutralize, any points on the sample that have accumulated positive charge. Positive secondary ion images from Teflon $(B)$ a well-known insulator, illustrate the effectiveness of charge compensation. Locating and identifying analytes on dry filter paper by using tandem mass spectrometry are also demonstràted.( (j Am Soc Mass Spectrom 1994, 5, 37-43)
\end{abstract}

I maging of biologic tissue by using secondary ion mass spectrometry (SIMS) is complicated by many factors. This is particularly true for mapping spatial distributions of targeted organic compounds in tissuc samples of a size comparable to those analyzed by autoradiography [1], that is, about $1 \mathrm{~cm}^{2}$. Biologic tissue is a very complex matrix, often yielding secondary ions at every mass. This chemical noise can overwhelm the secondary ion signal from compounds of interest [2]. Organic compounds of physiologic interest are often found at trace levels in tissue, and biologic samples suffer significant damage from excessive primary ion bombardment [3]. A further hinderance is that large tissue samples are usually very poor electrical conductors. Charge accumulates on the surface of an insulating sample during primary ion bombardment unless appropriate charge compensation measures are taken. Localized charging distorts secondary ion emission from the sample and severely perturbs the extraction field ion optics to the point that the secondary ion signal often completely disappears $[4,5]$. Sample charging has a deleterious effect on secondary ion transmission, because the sample surface is an integral part of the ion optical train of the microprobe secondary ion source. Any uncontrolled voltage in the ion optical train diminishes the performance of the mass spectrometer.

We previously reported [6] on the development of a high transmission large-sample secondary ion tandem mass spectrometry (SIMS/MS) microprobe for the analysis of complex organic samples. The design of the

Address reprint requests to Dr. Peter J. Todd, P.O. Box 2008, Building 5510, MS-6365, Oak Ridge National Laboratory, Oak Ridge, TN $37831-6365$
SIMS/MS microprobe was directed toward overcoming the problems of chemical noise, sensitivity, and sample damage associated with imaging biologic tissuc. Static SIMS (i.c., primary ion doses less than $10^{13}$ ions $/ \mathrm{cm}^{2}$ ) and tandem mass spectrometry (MS/MS) are used to minimize sample damage and reduce chemical noise, respectively. Computer-controlled ion optics and relaxed spatial resolution are used to enhance sensitivity. Previous reports on use of the instrument have been limited to analysis of conducting samples.

We now address the issue of secondary ion imaging of large insulating samples by using static SMS and MS/MS. A charge compensation scheme for imaging large biologic samples is subject to several constraints. The technique should neither cause sample damage nor interfere with secondary ion emission. Spatial resolution should not be sacrificed, and charge neutralization must be self-regulating to allow for variations in the rate of charging across the sample surface. Electrical conductivity across the surface of tissue samples can be very heterogeneous, particularly for rigorously prepared samples in excess of $1 \mathrm{~cm}^{2}$. A technique that is not self-regulating, when applied to heterogeneous samples, may cause negative charging in some areas while neutralizing positive charge in others. Thus, the method of charge compensation must permit the properties of the sample to control neutralization.

Many methods have been employed to mitigate sample charging for SIMS analysis, including use of a conducting grid $[5,7]$, coating the sample with a thin conducting layer [5, 8], and use of an electron flood gun $[9,10]$ to neutralize positive charge build-up. Hagenhoff et al. [11] have successfully implemented pulsed charge compensation by using a low energy 
electron gun and a pulsed extraction field for time-offlight (TOF) SIMS of insulating samples. With this method, the secondary ion extraction field is pulsed on during the very short primary ion beam pulse. After ions exit the source, the extraction field of the secondary ion source is zeroed. During the relatively long period in between primary ion pulses, a focused $10-\mathrm{eV}$ electron beam strikes the sample surface and neutralizes any accumulated positive charge. More recently, Appelhans et al. [12] introduced a technique that uses a negative primary ion beam in conjunction with an alternating-polarity secondary ion extraction field for a quadrupole mass analyzer. Any charge on the sample is neutralized by alternating between positive and negative ion extraction at the proper duty cycle. The polarity of sample charging reverses with extractionfield polarity when a negative primary ion beam is used. This approach requires no additional hardware or sample preparation, and has worked well for SIMS analysis of a variety of very insulating samples.

While each of these methods is appropriate for different types of samples and particular types of analyses, none is exactly suited for static STMS imaging of large $\left(\sim 1 \mathrm{~cm}^{2}\right)$ samples. Conducting grids are excellent references for verifying image fidelity; however, they interfere with imaging the sample of interest, contribute to signal background, and may introduce contamination onto the sample. Sample coating is widely used in dynamic SIMS, but coating the sample masks the surface and static SIMS can only sample the surface monolayer. Use of a focused electron flood gun may require extensive tuning for each sample and prior knowledge of sample composition. The presence of a secondary ion extraction field (typically $100 \mathrm{~V} / \mathrm{cm}$ or larger) complicates use of a low energy flood gun because the extraction field operates on electrons as well as ions. With the method of Appelhans et al. [12] (i.e., use of a negative primary beam with an alternating extraction field), the position of a focused primary ion beam on the sample will change when the extraction field polarity is reversed. A shift of a few hundred microns can be observed for a $6-\mathrm{keV}$ beam with even a modest extraction field (e.g., $100 \mathrm{~V} / \mathrm{cm}$ ). Unless the position of the primary beam is readjusted when the extraction field polarity is changed, areas of charging will not be neutralized. More important, the method is not self-regulating for possible variations in conductivity across a large sample; the proper duty cycle to neutralize sample charging at one point on the sample may not be appropriate at another position.

We have chosen to use a charge compensation scheme that combines appropriate features drawn from the techniques introduced by Hagenhoff et al. [11] and Appelhans et al. [12]. It is an extension of the electron flood gun technique, coupled with a periodic primary ion beam that is synchronized with the secondary ion extraction field. Low energy electrons $(5-10 \mathrm{eV})$ are allowed to flood the sample when the primary ion beam is turned off and the extraction field zeroed.
Electrons are attracted to areas that have accumulated positive charge and neutralize the sample in those areas. When the primary ion beam and secondary ion extraction voltage are turned on, the electrons are deflected away from the sample by the extraction field. Although this method is very similar to the technique used by Hagenhoff et al. [11] on a TOF SIMS instrument, there are several significant differences. TOF methods inherently require only very short primary ion beam pulses and much lower primary currents than quadrupole-based mass analyzers, which usually use continuous primary ion beams (i.e., the beam remains on while data are accumulated). The very low primary ion currents used in TOF techniques cause much less serious sample charging. In this regard, our technique is more similar to the periodic extraction field scheme reported by Appelhans et al. [12]. Also, the field of view of the TOF instrument used by Hagenhoff et al. [11] is $1 \mathrm{~mm}^{2}$, requiring use of a focused electron beam. Our SIMS/MS microprobe has a field of view in excess of $1 \mathrm{~cm}^{2}$; consequently, we use a diffuse "ribbonlike" spray of electrons to provide self-regulating charge compensation over the surface of large samples.

Although quadrupole mass analyzers are not normally thought of as pulsed sources, only minor modifications to the quadrupole-based SIMS/MS microprobe [6] were required to implement the charge compensation scheme described above. This is because, unlike most microprobes, which use capacitive coupling and continuous sawtooth waveforms to raster the primary beam, we discretely set the primary and secondary ion deflection plate voltages using digitalto-analog converters (DACs) [13]. Consequently, the primary ion beam can be turned off and the secondary ion extraction field simultaneously zeroed at each image pixel for any period of time without sacrificing spatial resolution or losing secondary ions from the sample.

\section{Experimental}

All data were obtained using a SIMS/MS microprobe, which has been described in detail earlier [6]. The microprobe consists of a wide-angle secondary ion source [13] interfaced with a triple quadrupole mass analyzer (Extrel, Madison, WI) and is capable of providing images of samples as large as $1 \mathrm{~cm}^{2}$. Three types of images can be obtained: (1) total ion images, by operating all three quadrupole mass analyzers in radiofrequency (rf) only mode; (2) secondary ion images [14], by operating the first quadrupole in massselected mode; and (3) MS/MS descendant ion images [6], by operating the triple quadrupole in tandem mass spectrometry mode. Except where noted in the text, experimental parameters for acquisition of images are listed in Table 1. Images are obtained by rastering the primary ion beam across the sample and measuring the intensity of transmitted secondary ions desorbed 
Table 1. Instrumental parameters for SIMS/MS microprobe

\begin{tabular}{ll}
\hline \multicolumn{1}{c}{ Parameter } & Value \\
\hline Primary ion species & $\mathrm{Cs}^{+}$ \\
Primary ion kinetic energy & $6.0 \mathrm{keV}$ \\
Primary ion current & 10.25 to 2.5) $\mathrm{nA}$ \\
Primary ion spot diameter & $100 \mu \mathrm{m}$ \\
Primary ion current density & $(2.5$ to 25$) \mu \mathrm{A} / \mathrm{cm}^{2}$ \\
Primary ion dose per image & $\left(0.2\right.$ to $2.01 \times 10^{12}$ ions $/ \mathrm{cm}^{2}$ \\
Primary ion duty cycle & $(3.5 \mathrm{~ms}) / 4.5 \mathrm{~ms})$ \\
Raster size & $1 \mathrm{~cm} \times 1 \mathrm{~cm}$ \\
Electron flood gun current & $200 \mu \mathrm{A}$ \\
Secondary ion energy & $10 \mathrm{eV}$ \\
Collision energy & $25 \mathrm{eV}$ \\
Collision pressure & $4 \times 10^{-5}$ torr \\
Conversion dynode potential & $-6.0 \mathrm{kV}$ \\
Multiplier potential & $-2.0 \mathrm{kV}$ \\
Data acquisiton rate & $50 \mathrm{kHz}$ \\
Data points per pixel & 128 \\
Pixel size & $50 \mu \mathrm{m}$ \\
Gain factor & $1 \times 10^{12} \mathrm{~A}^{-1}$ \\
\hline
\end{tabular}

Measured at the detector vacuum housing.

from each point on the sample. Lateral image resolution of $100 \mu$ is limited by the diameter of the primary ion beam spot and has been verified with grids [13]. Control of the myriad functions required to produce an image is performed by an 80286-based PC, using software developed at Oak Ridge National Laboratory.

Several modifications were made to the secondary ion source and data acquisition/control software, described in a previous report [13], to mitigate charging of nonconducting samples. The secondary ion source was modified by installing a $2-\mathrm{cm}$ length of $25-\mu \mathrm{m}$ diameter (50\% Re-50\% Mo) wire filament to produce low energy electrons. The filament was placed approximately $1.5 \mathrm{~cm}$ from the sample, behind a slotted aperture attached to the lens element nearest to the sample. The filament was nominally biased $-5 \mathrm{~V}$ relative to the slotted aperture assembly and the sample, which were both held at ground potential. (There was an additional $-4 \mathrm{~V}$ drop across the filament during operation due to the heater current.) Collimation of electrons by the slotted aperture thus created a "ribbon" beam of electrons, which passed over the sample in the absence of any additional electric fields. Since the filament potential was between $-5 \mathrm{~V}$ and $-9 \mathrm{~V}$, no point on the sample could develop a potential more negtive than $-9 \mathrm{~V}$. Electron emission current measured on the slotted aperture was regulated at nominally $200 \mu \Lambda$ and clectron current on the sample was measured to be approximately $200 \mathrm{nA}$ using a Faraday cup in place of the sample. The data acquisition/control software and electronics were also modified to allow the primary ion beam to be turned off, and the secondary ion extraction field independently zeroed, using TTL outputs from the computer. The primary ion beam is turned off by deflecting it away from a collimation aperture, and the extraction field is zeroed by nulling the secondary ion extraction voltage.
The general features of filament operation are shown in Figure 1.

Data for each image pixel were acquired in the following manner. The primary ion deflection plate voltages were set to direct the beam to a spot on the sample, and secondary ion deflection plates were adjusted for optimum secondary ion transmission. The secondary ion extraction voltage $(-400 \mathrm{~V})$ and primary ion beam were then tumed on. After an $800-\mu \mathrm{s}$ delay to allow for secondary ion transit through the mass analyzer, the transmitted amplified secondary ion current was sampled by a 12-bit analog-to-digital converter for approximately $2.6 \mathrm{~ms}$ (128 samples at $20-\mu$ intervals). The beam and extraction field were then turned off after a total ion beam exposure time at that spot of approximately $3.5 \mathrm{~ms}$. The average secondary ion signal was calculated for that pixel and the deflection plates were adjusted for the next spot, typically $50 \mu \mathrm{m}$ away. After a 1-ms delay, during which electrons flooded the sample, the process was repeated. Each image consists of 40,000 such pixels (200 $\times 200$ array), representing a sample area of approximately $1 \mathrm{~cm}^{2}$. The total dwell time per pixel was 4.5 ms, yielding a nominal image acquisition time of 3 minutes. Images are displayed using a nearly continu-

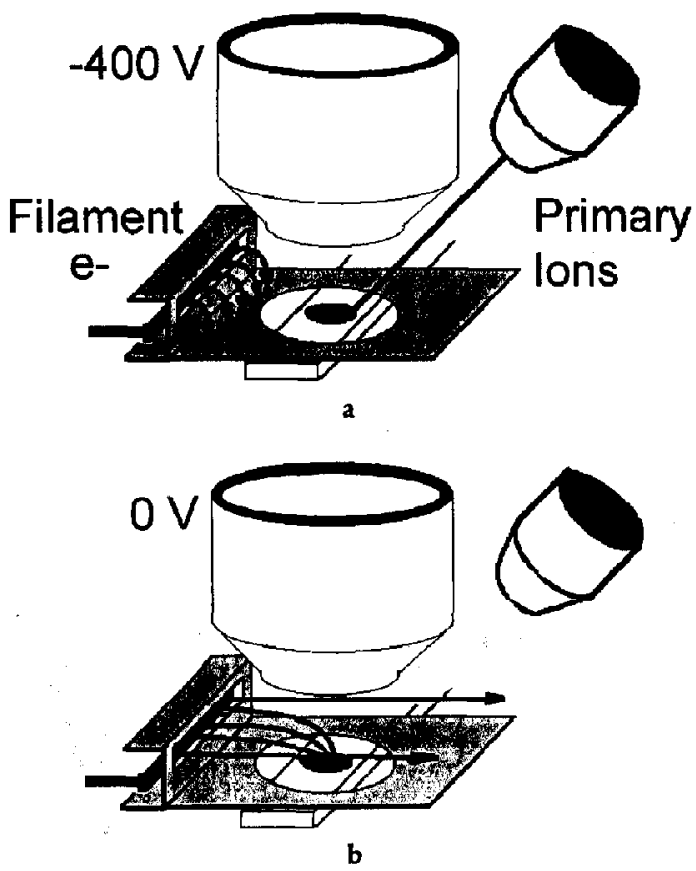

Figure 1. Charge compensation scheme. (a) During the period $(3.5 \mathrm{~ms}$ ) that the primary ion beam is turned on, the secondary ion extraction voltage $(-400 \mathrm{~V})$ deflects electrons away from the sample. (b) When the extraction voltage is zeroed, electrons are attracted to a charging spot on the sample. In the absence of sample charging, electrons pass over the sample. The primary beam is turned off during charge compensation period ( $1 \mathrm{~ms}$ ) to reduce primary ion damage. The process is repeated for each spot on the sample to produce an image. 
ous gray scale (256 shades), with "white" representing the most intense pixel in the image and "black" representing the least juterise pixel. The intensity saale for each image represents the output of the electron multiplier detector in amperes when divided by the gain factor in Table 1 . The total primary ion dose per image was 1 to 2 orders of magnitude below the static SIMS limit of $10^{1.3}$ jons $/ \mathrm{cm}^{2}$.

Mass spectra were obtained in a simular marner. The exception was that the position of the primary ion beam remained fixed while the quadrupole mass analyzer was scanned. Scanning the quadrupole mass andlyzer is accomplished using DAC's to step (the rf and dc levels of the quadrupole) across each mass peak in approximately $1 / 16 \mathrm{u}$ steps. The primary ion beam and extraction field were tumed off between cach step of the quadrupolc, allowing low eneryy electrons to flond the sample. A major difference between acquisition of images and mass spectra is that the static SIMS limit was exceeded at each spot from which mass spectra were obtained. When operating the microprobe in MS/MS mode, argon collision gas was leaked into the sccond quadrupole (operated in of unly mode) and the rise in background pressure was monitored by an ionization gauge at the detector housing. Secondary ions were accelerated from their nominal 10-eV energy to $25 \mathrm{cV}$ to increase efficiency of collision-induced dissociation.

biologic tissue is a poor reference for tesiting this technique, becaust (1) there are no appropriate standards, and (2) there may be other factors in addition to sample charging that inhibit secondary ion emission. Therefore, we have used simpler insulating samples as test cases. Three types of samples were used for the data presented here: Teflon:B; acetylcholine chloride on filter paper; actylcholine chloride on copper. The Teflon and filter paper samples were used as examples of insulating samples, whereas acetylcholine on copper was used to oblain reference mass spectra and tandem mass spectra of acetylcholine. A $4 \mathrm{~mm} \times 4 \mathrm{~mm}$ piece of Teflon tape $(80 \mathrm{\mu m}$ thick $)$ was placed onto a copper target using double-sided tape and was inserted into the vacuum system for aralysis without further preparation. A $6 \mathrm{~mm}$ diameter circular picee of filter paper (Whatman no. 5 qualitative) was similarly attached to a copper target. Then a $0.3-\mu \mathrm{L}$ drop of $10^{2} \mathrm{M}$ solution of acetylcholine chloride (Sigma Chemical Co., St. Louis, $\mathrm{MO}$ ) dissolved in distilled water was placed onto the center of the filter paper disk using an Eppendorf pipet. A similar $0.3-\mu \mathrm{L}$ drop of solution was placed directly onto the copper target at another sample position. The samples were allowed to air dry and were then inserted into the varuum system for analysis.

\section{Results and Discussion}

Figure 2 illustrates the effectiveness of charge compensation for insulators such as leflon. All three images

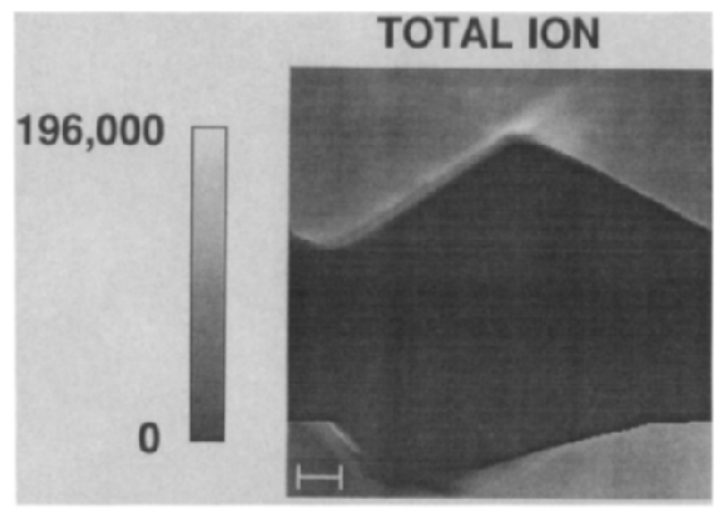

$\mathbf{a}$

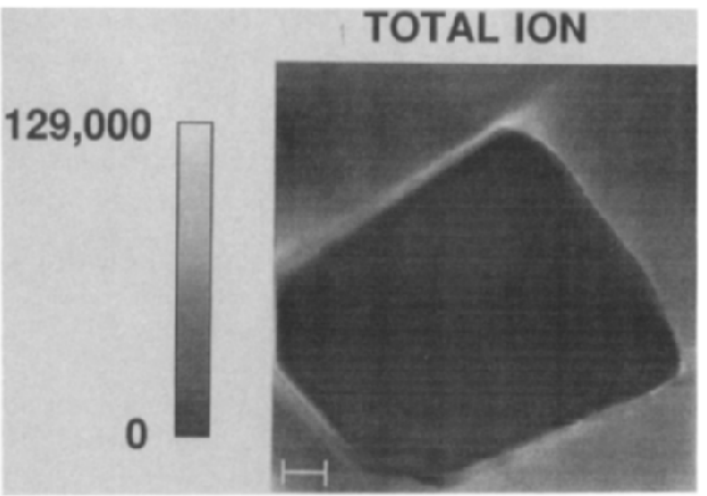

b

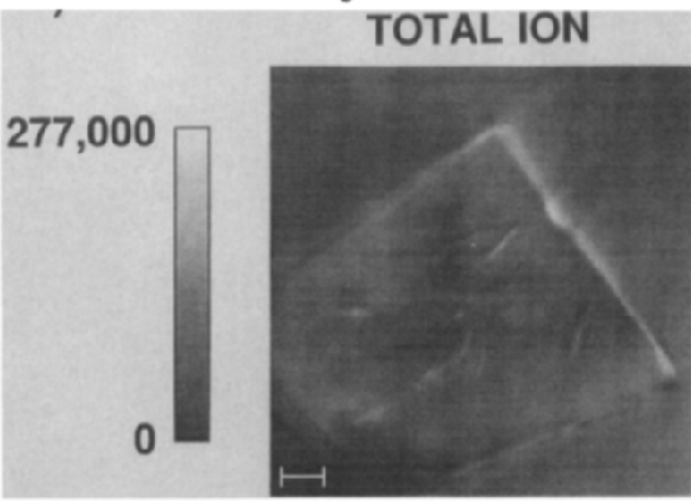

c

Figure 2. Serondary on images from Teflnn. (a) Total ion image without chasge compensation. (b) Tutal ion image with elistron flored gun only. \{c\} lotal inn image with plectron flood gun and periodic secondary ion cxiraction field. Siale: bars represent 0.5 $\mathrm{mm}$.

are total ion images and represent an area of $5 \mathrm{~mm} \times 5$ mum insterd of the nurmal 1 cm $\times 1$ cm listed in Table 1. The smaller image size better matched the size of the Teflon sample, which was located diagonally in the fiteld of view, Figure 2 a demonstrates the severity of sample charging in the absence of charge compensatiun (i.e. electron flood gun off and without a periodic secondary ion extraction field). Sample charging is 
manifested by the complete absence of secondary ion signal from the Teflon sample. In fact, the accumulated charge on the Teflon remained even after the primary beam had been moved to the copper target. This "memory effect" is evidenced by the absence of secondary ion signal in areas to the right and left of the Teflon as well (the primary ion beam was rastered from left to right, and by row from top to bottom). Figure $2 \mathrm{~b}$ was obtained by using only the electron flood gun for charge compensation and without periodically turning off the secondary ion extraction field. In this case, most electrons were deflected away from the sample by the extraction field and were not attracted to charged areas on the sample. Nonetheless, a reduction in sample charging is evident. The outline of the Teflon is better defined, indicating that secondary ions from the copper target were being collected. Secondary ions from the Teflon, however, were not detected except at the very periphery of the sample. Figure $2 \mathrm{c}$ demonstrates the utility of using the combination electron flood gun and periodic extraction field. Secondary ion emission from the Teflon was at approximately the same level as from the copper target as is evident from the gray scale. Topographical features of the Teflon, such as small creases or wrinkles, are noticeable. The bottom corner of the Teflon had curled up from the target, which resulted in the dark area at the bottom of the secondary ion image. Mass spectra from the Teflon showed major peaks consistent with the static secondary ion mass spectrum for polytetrafluoroethylene [15]. Mass spectra could not be obtained without charge compensation.

Figure $3 a$ is a mass spectrum obtained from the pure sample of acetylcholine chloride deposited directly onto the copper target. The acetylcholine molecular ion at $m / z 146$ is prominent, as well as other characteristic fragment peaks. The peak at $m / z 133$ is from implanted cesium from the primary ion beam. Figure $3 \mathbf{b}$ is the tandem mass spectrum of the acetylcholine $m / z$ 146, taken from an adjacent spot on the acetylcholine chloride sample. The base peak is $m / z$ 87, consistent with loss of trimethylamine from acetylcholine. From analysis of the pure sample, we chose the $m / z \quad 146$ secondary ion, and $m / z \quad 146 \rightarrow m / z 87$ fragmentation to be representative of acetylcholine for the purpose of mapping its spatial distribution on the filter paper.

Figure 4 demonstrates the ability to map heterogeneous distributions of compounds on insulating samples such as dry filter paper. Figure $4 d a$ and $b$ are total ion images of a 6-mm diameter circular piece of dry filter paper placed onto a copper target and spiked with $400 \mathrm{ng}$ of acetylcholine. Part $4 \mathrm{a}$ was obtained without charge compensation and part $4 \mathrm{~b}$ with charge compensation. Severe sample charging was observed without the electron flood gun and periodic extraction field, as evidenced by the oblong black region in the image. When charge compensation was used, however, secondary ion emission from the filter paper was

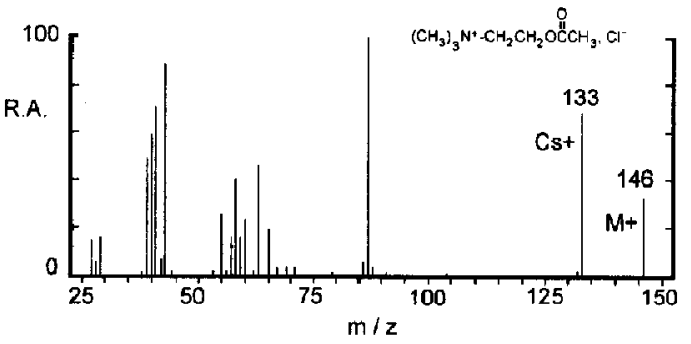

a

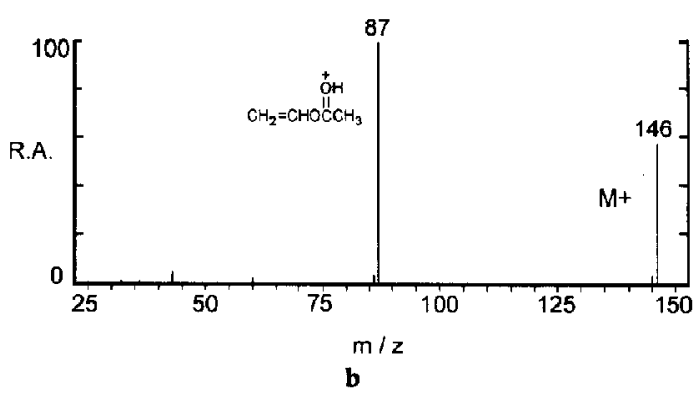

Figure 3. Reference spectra of acetylcholine chloride. (a) Secondary ion mass spectrum from acetylcholine chloride sample on copper target. Peak at $m / z^{3} 146$ is the acetylcholine secondary ion. (b) Tandem mass spectrum of acetylcholine $\mathrm{m} / \mathrm{z} 146$ ion. Fragmentation to $m / z 87$ is the most characteristic result of CID.

observed. The outline of the filter paper in Figure $4 \mathrm{~b}$ appears more as an ellipse than a circle because of the $45^{\circ}$ angle of the incident primary ion beam. The right edge of the filter paper had slightly separated from the sample holder producing a rough shadow effect on that side of the image. There is also a dark region to the left of the filter paper in Figure $4 \mathrm{~b}$, which we attribute to suppression of secondary ion emission because of reduction of the metal surface by the electrons. The electron filament was to the left of the sample (as viewed in Figure 4), yielding a higher density of low energy electrons on the left side of the sample, since the "ribbon" electron beam was not tightly collimated. We have observed a similarly slight suppression of secondary ion signal from other metal surfaces when the electron gun was used. Secondary ion emission from organic conductors does not appear to be significantly affected by the electron beam. We have verified this by taking images of organic compounds deposited neat onto metal targets both with and without the electron beam. While secondary ion signal from the metal surface is lower when using charge compensation, secondary ion yields from the organic samples do not change significantly. Although there is the possibility that use of the electron beam could suppress secondary ion emission from some organic samples, a net increase in secondary ion yield from insulating samples justifies use of the technique. There is also the possibility that the low energy electrons could chemically modify the sample, although we have seen no evidence of this effect. 


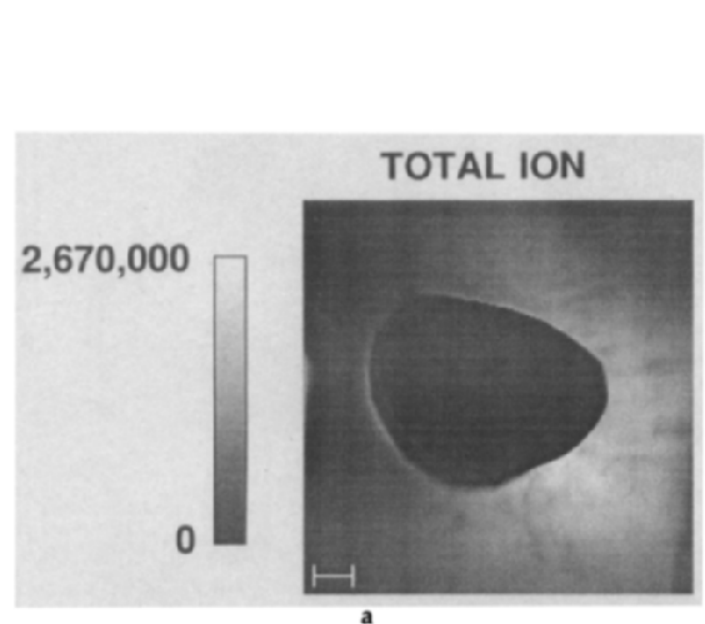

a
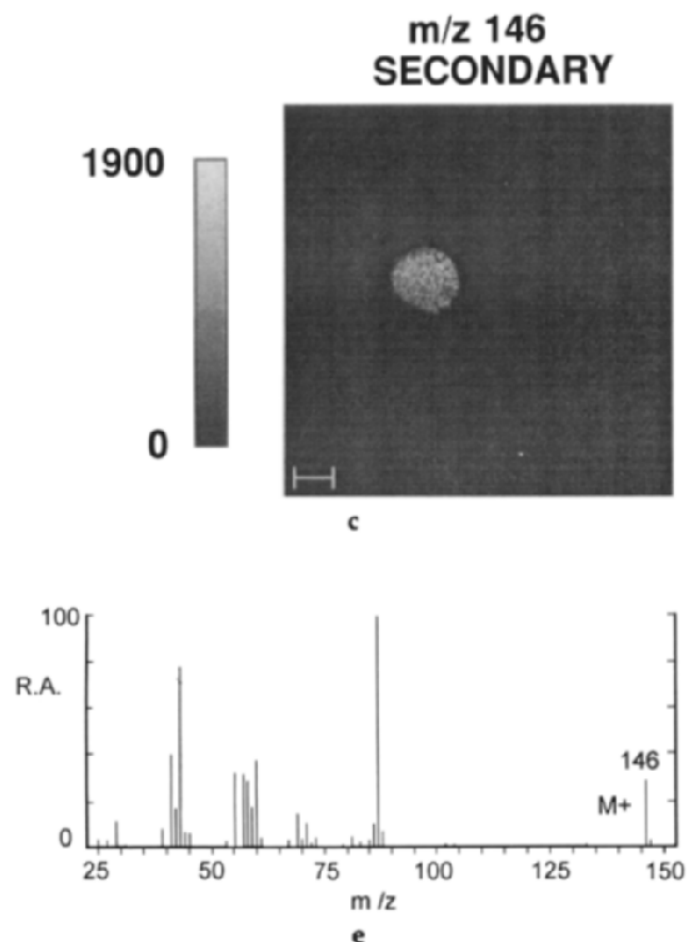

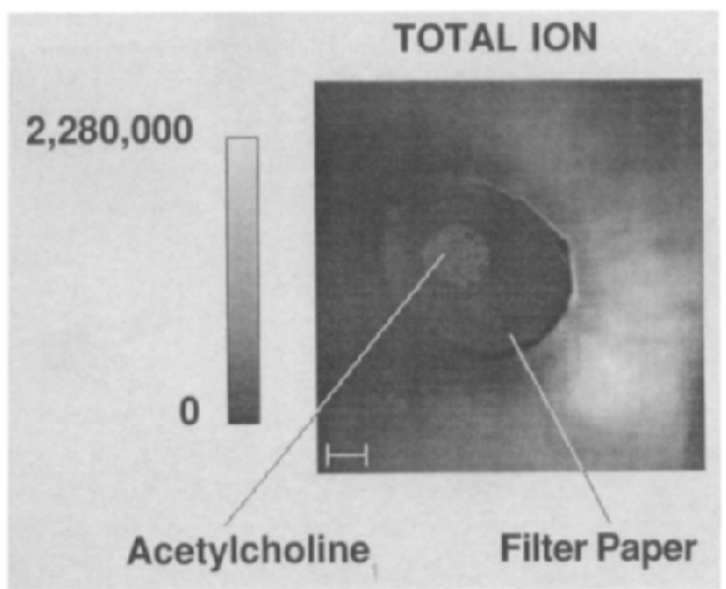

b

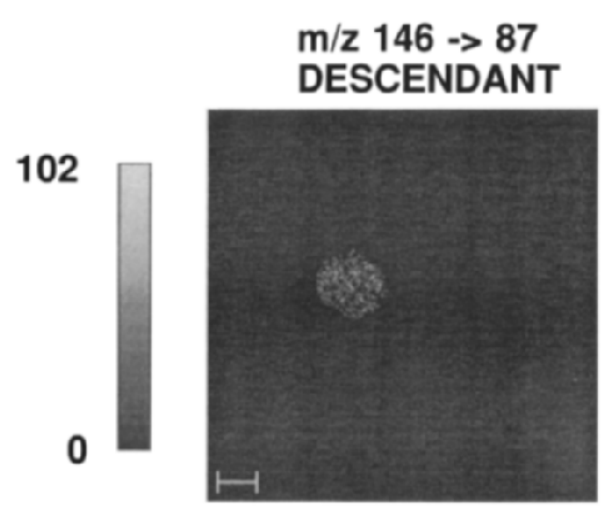

d

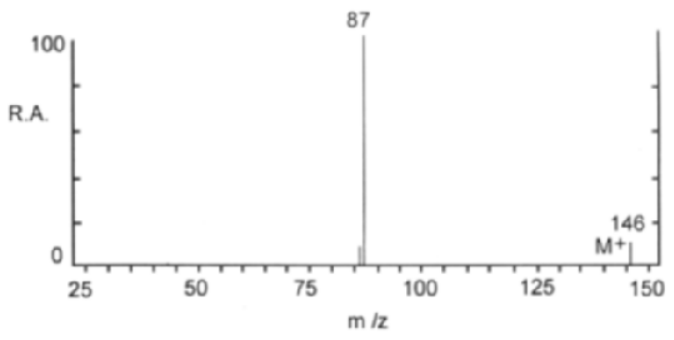

$f$

Figure 4. Analysis of actykloline on dry filtur puper. (a) Total ion image without charge compensation. (b) Tatal ion image wijth electron flind gun and periodic secondary iun extractum field. (c) Mass resolved secondary ion image of $m / 1$ 146, characteristic secondary inn of acetylcholine. (d) Lescendant ion image of the $m / z 146 \cdot m / z 87$ MS/MS transition, characteristic uf acelulchuline. (e) Secondury ion mass spectrum from acetylcholine chloride sample on filter paper. (f) Tandem mass spectnum of acetylcholine $m / z$ ith ion from filter paper. Sale bas in images represent $1.0 \mathrm{~mm}$.

Figure $4 \mathrm{c}$ is the mass-resolved secondary ion image of $m / z \quad 146$. The most intense region of secondary ion emission was from the center of the filter paper where acetylcholine was placed. Figure $4 \mathrm{~d}$ is the MS/MS descendant ion $(m / z 146, m / z 87)$ image from the same sample. Il confirms both the identity and location of acetylcholine. Quadrupole 1 was set to transmit only secondary ions with $m / z 146$ and quadrupole 3 was set to transmit only ions with $m / z 87$ following CID of the $m / z 146$ ions with argon in the collision cell, 
quadrupole 2. We have found that SIMS/MS imaging (i.e., imaging with MS/MS) is often necessary to obtain accurate spatial distributions of targeted organic analytes; chemical noise can at times overwhelm a secondary ion image, leading to inconclusive or incorrect interpretation regarding the actual distribution of analyte [6]. Use of MS/MS; however, reduces overall sensitivity [16]. In this case, the acetylcholine spot was approximately $2 \mathrm{~mm}$ in diameter and contained a total of $400 \mathrm{ng}$ of acetylcholine, so that extremely high sensitivity was not necessary. Each (100- $\mu$ m diameter) image pixel, however, contained only an average of 1 ng of acetylcholine, which had diffused throughout the thickness of the filter paper. Figure $4 e$ and $f$ are a mass spectrum and tandem mass spectrum, respectively, from the acetylcholine spot on the filter paper. They are qualitatively the same as Figure $3 a$ and $b$, with the notable absence of the $\mathrm{Cs}^{+}$peak in Figure 4e. Again, no mass spectra were obtainable from the insulating sample without charge compensation.

Unsuccessful attempts were made to obtain negative secondary ion mass spectra and images from both the Teflon and filter paper samples. Since serondary electron emission, which is typically an order of magnitude higher than secondary ion emission [5], is not suppressed with negative ion extraction, sample charging is exacerbated in this mode. We were unable to increase the charge compensating electron current sufficiently to neutralize these samples effectively with the present apparatus. Future work will be directed toward adaptation of this technique to analyze negative secondary ions from insulators.

\section{Conclusions}

This technique appears to be self-regulating over the surface of large samples, and little or no change in operational parameters is required from sample to sample. Furthermore, we have found that this technique satisfactorily meets other criteria for large sample imaging: the low energy electrons do not cause further sample damage; they do not appear to inhibit organic secondary ion emission; the technique does not degrade spatial resolution. The capability to routinely map distributions of organic compounds on the surfaces of both conducting and nonconducting samples significantly advances our efforts to analyze drugs and their metabolites in biologic tissue. Spatial mapping of organic compounds in other large samples (e.g., organic species of geochemical interest in coal samples) should also be possible. The ability to image large insulating samples greatly reduces sample preparation time and complexity. Large samples are much easier to handle, and minimal preparation reduces potential loss of sample integrity from the excessive handling inherent to complex preparation procedures.

\section{Acknowledgments}

The SIMS/MS microprobe is on loan from the Biomedical and Instrumentation Engineering Program, National Center of Research Resources, National Institutes of Health. I'his work was supported by the National Institutes of Health grant no. RO1 GM41617 and by the U.S. Department of Energy, Office of Basic Energy Sciences, under contract DE- $\Lambda$ C05-84OR21400 with Martin Marietta Energy Systems, Inc.

\section{References}

1. Herkenham, M.; Little, M. D.; Bankiewicz, K.; Yang, S.-C.; Markey, S. P.; Johannessen, J. N. Neuroscience, 1991, 40, 133.

2. Gillen, G; Hues, S. M. J. Am. Soc. Mass Spectrom. 1993, 4, 419.

3. Gillen, G.; Simons, D. S.; Williams, P. Anal. Chem. 1990, 64, 2122.

4. Werner, H. W.; Morgan, A. E. J. Appl. Phys. 1976, 47, 1232.

5. Benninghoven, A.; Rudenauer, F. G.; Werner, H. W. Secondary Ion Mass Spectrometry; Wiley: New York, 1987; pP 881-899.

6. Todd, P. J.; Short, R. T.; Grimm, C. C.; Holland, W. M.; Markey, S. P. Anal. Chem. 1992, 64, 1871.

7. Werner, H. W.; Warmoltz, N. I. Vuc. Sci. Techrol. 1984, 2, 726.

8. Slozdian, G. Ann. Phys. 1964, 9, 591.

9. Briggs, D.; Wootton, A. B. SIA, Surf. Interface Anal. 1982, 4, 109

10. Eccles, A. J.; Vickerman, J. C. Vac. Sci. Technol., A 1989, 7, 234.

11. Hagenhoff, B.; van Leyen, D.; Niehaus, E.; Benninghoven, $A$. I. Vac. Sci. Technol, A 1989, 7, 3056.

12. Appelhans, A. D.; Dahl, D. A.; Delmore, J. E. Anal. Chem. $1990,62,1679$.

13. Grimm, C. C.; Short, R. T.; Todd, P. J. J. Am. Soc. Mass Specirom. 1991, 2, 362.

14. Kriger, M. S.; Cook, K. D.; Short, R. T.; Todd, P. J. Anal. Chem. $1992,64,3052$.

15. Static SIMS Handbook of Polymer Analysis; Perkin-Elmer Corp., 1991; 138.

16. Todd, P. J.; Short, R. T. Procedings of the 40th Annual ASMS Conference on Mass Spectrometry and Allied Topics; Washington DC, May-June 1992; p 1961. 